\title{
Risikoreduktion durch Fettsäure-Switch
}

Fragestellung: Welchen Effekt hat gesunde Ernährung bei neu diagnostizierten (Prä-)Typ-2-Diabetikern auf den Verlauf?

Hintergrund: Eine gesunde Ernährung ist Basis für die Diabetesprävention und ein effektives Selbstmanagement bei Typ-2-Diabetes. Sie hilft die glykämische Last zu verringern und kann pleiotrope Effekte im Hinblick auf eine verbesserte Insulinsensitivität und Prävention von kardiovaskulären Erkrankungen erzeugen.

Patienten und Methodik: 509 Personen, die im Rahmen eines Screening-Programms mit einer gestörten Glukosetoleranz, gestörter Nüchternglukose oder neu diagnostiziertem Typ-2Diabetes auffällig wurden, wurden im Rahmen der ADDITION Studie (Anglo-Danish-Dutch Study of Intensive Treat-

Maindal HT, Toft U, Lauritzen T, Sandbæk A. Three-year effects on dietary quality of health education: a randomized controlled trial of people with screen-detected dysglycaemia (The ADDITION study, Denmark).

Eur J Public Health. 2012 Jun 13. [Epub ahead of print] ment in People With Screen Detected Diabetes in Primary Care) rekrutiert und dann in eine Kontrollgruppe und eine Interventionsgruppe mit einem Ernährungs-SchulungsProgramm randomisiert.

Die Interventionsgruppe wurde einem 12-wöchigen Programm zugeteilt, das aus 8 Gruppen-Sessions und 2 Einzelsitzungen - insgesamt 18 Interventionsstunden - bestand. Der Fokus in dem Programm lag darin, den Teilnehmern im Rahmen ihres eigenen Selbstmanagements zu helfen einen gesünderen Ernährungsstil in ihrem

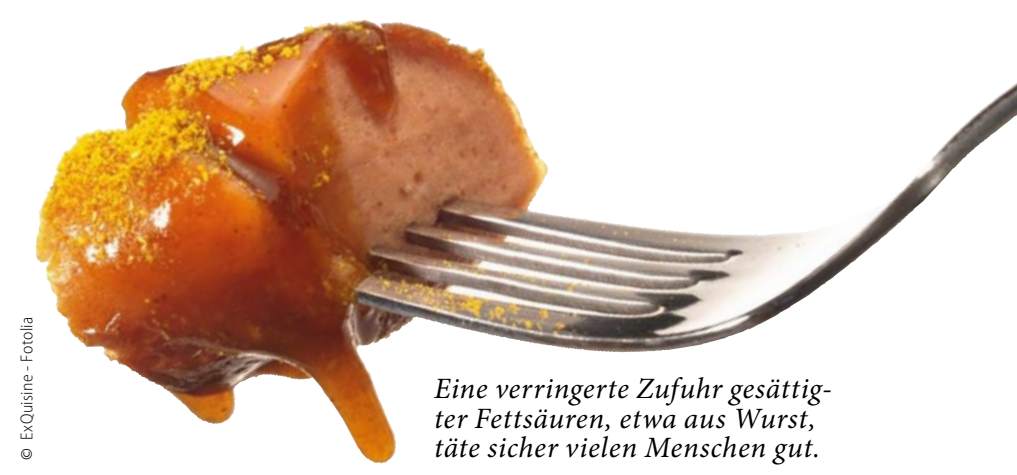

Alltag umzusetzen. Die Ernährungsqualität wurde mithilfe des Dietary Quality Score und einer Baseline evaluiert und nach 3-jährigem Follow-up analysiert. 444 Patienten konnten ausgewertet werden.

Ergebnisse: Nach 3-jährigem Verlauf zeigte sich, dass die Teilnehmer in der Interventionsgruppe ihren Ernährungslebensstil signifikant und nachhaltig verändert hatten. Der Verbrauch von gesättigten Fettsäuren nahm stark ab, der von ungesättigten Fettsäuren nahm signifikant zu. Kein Unterschied zeigte sich hinsichtlich des Obst- und Gemüsekonsums, der in beiden Gruppen zugenommen hatte.

Schlussfolgerungen: Das Interventionsprogramm war geeignet, um den Ernährungsstil nachhaltig über 3 Jahre zu beeinflussen. Herausragend war dabei die signifikante Reduktion der Aufnahme von gesättigten Fettsäuren.

\section{- Kommentar von Prof. Dr. med. Peter Schwarz}

\section{Wir sollten Prävention und Versorgung verknüpfen}

Wir beschweren uns sehr schnell darüber, dass unsere Patienten nicht so gesund leben, wie wir es ihnen empfehlen. Häufig ernähren wir uns selbst auch nicht so gesund, wie wir es anderen empfehlen. Wie kann man es also schaffen, einen nachhaltigen gesunden Ernährungsstil zu unterstützten? Das wurde als Teil der ADDITION-Studie untersucht. Und eine gut strukturierte Schulung konnte bei Neudiagnose einer gestörten Glukosetoleranz oder eines Typ-2-Diabetes nachhaltig helfen, den Ernährungsstil der Betroffenen über 3 Jahre positiv zu beeinflussen. Durch die Reduktion der Aufnahme von gesättigten Fettsäuren und den vermehrten Konsum von ungesättigten Fetten kam es zu einer 40\%igen Risikoreduktion hinsichtlich des Diabetesrisikos. Das ist ein starkes Signal! Es ist auch nicht überraschend. Ich finde die Studie interessant, da hier auf bestehender deutscher gesetzlicher Grundlage ( $\$ 20$ SGB 5) Interventionen umgesetzt wurden, die diesen nachhaltigen Effekt haben. Bedauerlicherweise gilt dieser Paragraf nur für die Primärprävention, in dieser Studie wurden aber auch Patienten untersucht. Wenn wir es schaffen, die Bereiche Prä- vention und Versorgung in Zusammenarbeit mit Krankenkassen zu verknüpfen, dann bietet sich die Möglichkeit, bei neu diagnostizierten Patienten die Präventionskompetenz in der Ernährungsschulung anzuwenden und die Effekte, die hier gezeigt wurden, für sie zu realisieren. Wir können erwarten, dass das mit Gewichtsreduktion und einer Prävention der Erkrankung als auch mit einer signifikanten Verbesserung des Outcomes der Erkrankung einhergeht

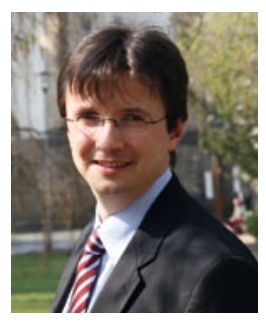

Prof. Dr. med. Peter E.H. Schwarz

Department of Medicine III

Prevention and Care of Diabetes

University of Dresden

Fetscherstr. 74

01307 Dresden

peter.schwarz@uniklinikum-dresden.de 